\title{
Encapsulation Using Plant Proteins: Thermodynamics and Kinetics of Wetting for Simple Zein Coacervates
}

\author{
Xiufeng Li, Philipp Erni, Jasper van der Gucht, and Renko de Vries* \\ Cite This: ACS Appl. Mater. Interfaces 2020, 12, 15802-15809 \\ Read Online
}

ABSTRACT: Traditionally, complex coacervates of oppositely charged biopolymers have been used to form coatings around oil droplets for encapsulation of oil-soluble payloads. However, many proteins can form coacervates by themselves under certain conditions. Here, we revisit the well-known simple coacervates of prolamins such as zein in mixed solvents to explore whether they can be used for plant-based encapsulation systems. We show that, for zein in mixed water/ propylene glycol (PG) solvents, we can encapsulate limonene droplets but only under specific conditions. We illustrate that this limitation is due to the very different physical properties of the simple zein coacervates as compared to those of the more extensively studied complex coacervates. Droplets of simple coacervates of zein can carry a significant net charge, whereas complex coacervates are usually close to being charge-balanced. In particular, we demonstrate that the

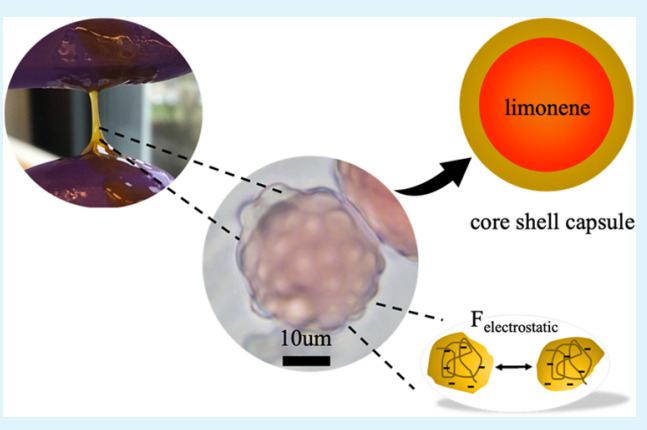
spreading of zein coacervates at the interface of the droplets is thermodynamically favorable due to their extremely low interfacial tensions in both the dispersed $(\sim 0.24 \mathrm{mN} / \mathrm{m})$ and oil phases $(\sim 0.68 \mathrm{mN} / \mathrm{m})$, but the kinetics of coacervate droplet deposition and the interactions among coacervate droplets that oppose coacervate droplet coalescence are highly $\mathrm{pH}$-dependent, leading to a sharp $\mathrm{pH}$ optimum (around $\mathrm{pH} 8$ ) for capsule formation.

KEYWORDS: plant protein, simple coacervation, encapsulation, interfacial tension, wetting

\section{INTRODUCTION}

Delivery and release of active ingredients by microcapsules is of great interest among others for pharmaceutical, ${ }^{1}$ personal care, ${ }^{2}$ and food applications. ${ }^{3}$ Many strategies are available to formulate microcapsules. For instance, charged actives can be encapsulated by forming micelles with oppositely charged polymers, ${ }^{4}$ and lipophilic molecules can be stabilized by surfactants or formulated as Pickering emulsions. ${ }^{5}$ In some cases, the actives have also been covalently attached to the carrier materials. $^{6}$

A classic technique for the microencapsulation of relatively hydrophobic active ingredients is by coating oil droplets with a thick layer of coacervate (the polymer dense phase of a liquidliquid phase separated polymer system), which is subsequently cured to form a shell. ${ }^{7,8}$ This is an attractive technology since coacervation can bring large amounts of polymers to interfaces and can produce fine capsules with a very high loading efficiency $(40-90 \%){ }^{9}$

For both food and nonfood applications, there is a strong interest in microencapsulation techniques that would rely on just abundantly available plant biopolymers and still have competitive performance. For most nonfood applications, the required performance objectives can currently only be achieved using synthetic polymers. On the other hand, for food applications, the dominant approach to (coacervate-based) microencapsulation still involves the use of animal proteins. A case in point are the well-studied complex coacervates of weakly charged polysaccharides such as gum arabic with animal proteins such as gelatin or whey protein. ${ }^{10}$

Many of the most abundant plant storage proteins, such as the prolamins and globulins in leguminous plants, are poorly soluble in water. ${ }^{11}$ Additionally, the production of industrial plant protein concentrates and isolates often involve processing steps that lead to irreversible denaturation of (part of) the proteins. ${ }^{12}$ As a consequence of these factors, it is difficult to use proteins from these sources to formulate complex coacervates that can be used for microencapsulation. Previous studies show that some purified plant proteins (soy, pea, and wheat proteins) or certain fractions of them can be formulated into complex or simple coacervates for encapsulation. ${ }^{13}$ Identifying suitable processing approaches to use the less soluble plant storage proteins for microencapsulation purposes would not only be interesting from the point of view of sustainability but also because their more hydrophobic nature may translate into better barrier properties.

Received: November 14, 2019

Accepted: March 2, 2020

Published: March 2, 2020 
One approach that has been quite extensively studied to use the less soluble plant storage proteins as physical barriers around oil droplets in oil-in-water emulsions is to create colloidal plant protein particles and use these to formulate Pickering emulsions. ${ }^{14,15}$ This requires careful tuning of the hydrophobic/hydrophilic balance of the particles, which has been achieved to some extent by also incorporating more hydrophilic biopolymers. ${ }^{16}$ For example, the main storage protein of corn, zein, like other prolamins, is soluble in aqueous ethanol binary solvents due to its unique amino acid composition, ${ }^{17}$ and this solubility behavior can be exploited to create colloidal zein particles by precipitation into aqueous (anti)solvents. ${ }^{18}$ Velikov and co-workers reported that zein colloids, synthesized by such an anti-solvent precipitation method, can stabilize soybean oil-water interfaces. ${ }^{5}$ Furthermore, colloidal stability of the emulsions itself could be improved by coating zein colloids with gum arabic. ${ }^{19}$ However, Pickering stabilized emulsions usually rely on a large interfacial energy barrier between the oil and water phase plus a precisely tuned amphiphilicity of the particles. This implies that, with this method, it is still challenging to encapsulate less hydrophobic and somewhat smaller molecules $\left(M_{\mathrm{w}} \approx 100-\right.$ $250 \mathrm{~g} / \mathrm{mol}$ ). Moreover, interfacial layers formed by discrete solid particles remain porous for molecular-size payloads even at the maximum interfacial packing fraction, limiting their suitability as an interfacial permeation barrier.

In the past, it has been found that some plant proteins, under certain conditions, exhibit liquid-liquid phase separation into a protein dense (often called coacervate) and a protein dilute phase (often called excess phase). Simple coacervation is typically found in a narrow range of solution conditions in between one- and two-phase regions where dilute phases coexist with precipitates. For prolamins in mixed solvents, coacervates are found over a narrow range of solvent compositions. ${ }^{17}$ Similarly, coacervation of leguminous globulins occurs for a narrow range of $\mathrm{pH}$ and salt concentrations. $^{12}$

Therefore, a way forward in using the more hydrophobic plant storage proteins for encapsulation purposes might be to exploit the simple coacervates that they form. While encapsulation by complex coacervates has been studied extensively, encapsulation using simple coacervates has hardly been studied. Encapsulation of oil droplets by simple coacervates of soy glycinin has been discussed previously where core shell capsules were shown to be formed by slowly inducing coacervation. ${ }^{20}$

Complex and simple coacervate droplets however have intrinsically very different physical properties that will translate into a very different behavior in the encapsulation process, and so far, these differences have not been elucidated. For example, at the optimal mass ratio and $\mathrm{pH}$, at which the yield of coacervate is maximal, complex coacervate droplets are nearly neutral, ${ }^{10}$ so once they are formed, they have a tendency to aggregate and coalesce. On the other hand, simple coacervate droplets can carry net charges such that they can be kinetically stable and resist coalescence. Also, due to their hydrophobic nature, simple plant protein coacervates may tend to have higher protein contents and hence can have higher viscosities, which may also influence the kinetics of the encapsulation process.

To investigate this issue, we here study the thermodynamics and kinetics of wetting for simple coacervates of zein and demonstrate the encapsulation of a low molecular weight hydrophobic molecule, limonene. Simple coacervation of zein is well known to occur in ethanol-water binary solvents, ${ }^{17}$ but in many cases, ethanol will not be a suitable cosolvent in the encapsulation processes because it is miscible with many low weight hydrophobic molecules to be encapsulated, such as limonene. We therefore investigate propylene glycol as a cosolvent and identify the condition of zein coacervation in mixed propylene glycol-water solvents. Coacervation on oil droplets is induced by slowly adding water, coming from the one-phase region at high propylene glycol.

We show that, for a broad range of conditions, zein coacervate droplets thermodynamically want to wet the interface with the limonene, but that for most conditions, they are kinetically prevented from doing so. We also show, however, that special conditions do exist for which the formation of thick zein coacervate layers around the limonene droplets is kinetically possible.

\section{MATERIALS AND METHODS}

Materials. Zein from corn (Z-3625), propylene glycol (W294004), (R)-(+)-Limonene (97\%, 183164), Nile Red (738567-3), and Oil Red O (O0625) were bought from Sigma-Aldrich. Hydrochloric acid $(1$ and $0.1 \mathrm{~N})$ and sodium hydroxide solutions (1 and $0.1 \mathrm{~N}$ ) were used to adjust $\mathrm{pH}$ and were from Merck. Ethanol absolute (AR) was purchased from Biosolve BV. Milli-Q water was used in all experiments.

Phase Diagram. Eight stock solutions (with different $\varphi_{\mathrm{PG}}$ ) were prepared as follows: for each stock solution, $2 \mathrm{~g}$ of zein was dissolved in $160,140,130,120,110,100,60$, and $20 \mathrm{~mL}$ of PG (since it is already known that zein is soluble in pure PG or $80 \% \mathrm{v} / \mathrm{v}$ PG with water $\left.^{21}\right)$. Next, $40,60,70,80,90,100,140$, and $180 \mathrm{~mL}$ of water were gradually added to the zein solutions, respectively. In this way, the final volume of each stock solution was $200 \mathrm{~mL}$ with a constant zein concentration of $1 \% \mathrm{w} / \mathrm{v}$. The $\mathrm{pH}$ of the stock solutions was adjusted to $\mathrm{pH} 10.0,8.0,5.3$, and 2.6 using small amounts of hydrochloric acid $(1$ and $0.1 \mathrm{~N})$ and sodium hydroxide solutions $(1$ and $0.1 \mathrm{~N})$. The phase diagram is constructed based on both microscopic images and macroscopic visual observation of the sample vials after spinning at $4500 \mathrm{rpm}$ for $30 \mathrm{~min}$. From optical microscopy, samples are considered to be in the two-phase coacervate region if they show transparent and spherical microscopic droplets. Samples that are completely clear in optical microscopy are considered to be in the one-phase region. Samples that show irregular microscopic particles and aggregates are classified as being in the two-phase precipitated region. Macroscopic visual observation of sample vials after centrifugation is required to be consistent with the microscopic observation: samples in the one-phase region have no sediment and a clear supernatant. Samples in the two-phase coacervate region have a viscous liquid phase, and samples in the two-phase precipitated region have a powdery, solid precipitate.

Coacervate Yield and Protein Fraction. Solutions with $2 \mathrm{~g}$ of zein dissolved in $40 \mathrm{~mL}$ of $80 \% \mathrm{v} / \mathrm{v}$ PG solvent were prepared. The $\mathrm{pH}$ of the zein solutions was adjusted to different values ( $\mathrm{pH} 2.92$, $3.47,4.02,4.53,5.17,6.07,6.46,7.13,7.63,8.00,8.50,9.11,9.48$, and 9.96) using hydrochloric acid ( 1 and $0.1 \mathrm{~N})$ and sodium hydroxide solutions $(1$ and $0.1 \mathrm{~N})$. Next, $10 \mathrm{~mL}$ of water was added to each solution (this dilution changes the $\mathrm{pH}$ by no more than \pm 0.1 ), and all samples were centrifuged at $4500 \mathrm{rpm}$ for $30 \mathrm{~min}$ at room temperature to accelerate the sedimentation of the zein coacervate. The zein coacervate was weighed after removing the supernatant, and the samples were freeze-dried to remove water. To completely remove PG, freeze-dried samples were redispersed in $10 \mathrm{~mL}$ of $80 \% \mathrm{v} / \mathrm{v}$ ethanol with water, $30 \mathrm{~mL}$ of water was added to precipitate zein into colloidal particles, and $\mathrm{NaCl}$ was added until a final concentration of $100 \mathrm{mM}$, which leads to aggregation of the colloidal zein. Next, the supernatant was removed using centrifugation. The process was repeated at least three times to thoroughly remove PG. Finally, samples were freeze-dried. The coacervate yield is the final weight 
(after freeze drying) divided by the initial amount of zein $(2 \mathrm{~g})$, and the mass fraction of protein in the coacervate is determined by dividing the final weight (after freeze drying) by the measured coacervate weight.

Rheology. Rheological measurements were performed with an Anton Paar rheometer 501 equipped with a Peltier element for temperature control. The temperature was controlled at $20{ }^{\circ} \mathrm{C}$. A 25 $\mathrm{mm}$ plate-plate geometry with a gap size $0.5 \mathrm{~mm}$ was used. The coacervate samples with different $\mathrm{pH}(\mathrm{pH} 7,8,9$, and 10) were prepared as described previously. In $65 \% \mathrm{v} / \mathrm{v}$ PG, the supernatant was decanted after centrifugation and coacervate samples were transferred onto the plate. We measured viscosity of the coacervate samples as a function of a shear rate from $10^{-6}$ to $100 \mathrm{~s}^{-1}$ (viscosity was not measurable at shear rates around $5 \mathrm{~s}^{-1}$ due to wall slip). We performed a gentle pre-shear step (from $10^{-6}$ to $1 \mathrm{~s}^{-1}$ ) and waited for $5 \mathrm{~min}$ before all measurements to make samples as homogeneous as possible and let samples stick to the plates. The shear stress was monitored to confirm that there is no shear history effect on the viscosity measurements.

Zeta Potential. Zeta potential was measured for zein colloids as a function of $\mathrm{pH}$ using a Zetasizer NanoZS apparatus (Malvern Instruments, UK) equipped with a $4 \mathrm{~mW} \mathrm{He}-\mathrm{Ne} 88$ ion laser $(\lambda=633$ $\mathrm{nm})$. The zein colloids were prepared by an antisolvent precipitation method. The hydrodynamic size of the zein colloids was determined using dynamic light scattering, and a diameter was found to be around $100 \mathrm{~nm}$. The zein colloids were dispersed in Milli- $\mathrm{Q}$ water, and various $\mathrm{pH}$ values (from $\mathrm{pH} 3.00$ to 9.88) were achieved and adjusted by hydrochloric acid $(1$ and $0.1 \mathrm{~N})$ and sodium hydroxide solutions ( 1 and $0.1 \mathrm{~N})$. Each sample was measured three times at $20^{\circ} \mathrm{C}$, and the Smoluchowski equation was used for converting measured mobilities to zeta potentials.

Pendant Drop Measurements. Interfacial tensions between limonene and $65 \% \mathrm{v} / \mathrm{v}$ PG at $\mathrm{pH} 7,8,9$, and 10 were measured with a drop tensiometer (Tracker from Teclis) using a reverse needle configuration. For all measurements, the droplet area is constant at 15 $\mathrm{mm}^{2}$. Each sample was measured 20 times with a data acquisition rate of one measurement per second.

Surface Tension Determination via Capillary Thinning. We obtained the interfacial tensions of coacervate in its excess phase and in the oil phase using capillary thinning experiments, which have been previously described by Dardelle and Erni. ${ }^{8}$ Capillary thinning experiments were performed with the zein coacervate prepared at $\mathrm{pH} 7$ as described above. After centrifugation at $4500 \mathrm{rpm}$ for $30 \mathrm{~min}$, we reinjected the coacervate in its supernatant or limonene on a glass surface, and the thinning dynamics of filaments were recorded by a microscope camera and analyzed with ImageJ. For coacervate filaments at $65 \% \mathrm{v} / \mathrm{v}$ PG $(\mathrm{pH} 7)$ or limonene, we analyzed five samples by measuring the neck width as a function of time.

Microcapsule Preparation. Typically, $2 \mathrm{~g}$ of zein was dissolved in $80 \mathrm{~mL}$ of $80 \% \mathrm{v} / \mathrm{v}$ PG $(65 \mathrm{~mL}$ of PG $+15 \mathrm{~mL}$ of water $)$ in a 250 $\mathrm{mL}$ beaker, and $1 \mathrm{~mL}$ of limonene with Oil Red O pre-dissolved (for staining) was added to be encapsulated. A magnetic stirrer was used to prevent limonene droplets from coalescing. The stir rate was typically at $430 \mathrm{rpm}$ unless specifically mentioned. Hydrochloric acid $(1$ and $0.1 \mathrm{~N})$ and sodium hydroxide solutions $(1$ and $0.1 \mathrm{~N})$ were used to adjust the solutions to the desired $\mathrm{pH}(\mathrm{pH} 3,4,5,7,8,9$, and $10)$. Then, $20 \mathrm{~mL}$ of water was dropwise added by a syringe pump (1 $\mathrm{mL} / \mathrm{min}$ ). $\mathrm{pH}$ changes due to the addition of water were less than $\pm 0.1 \mathrm{pH}$ units.

CLSM (Confocal Laser Scanning Microscopy). We used CLSM to map the distribution of limonene droplets within capsules. Fluorescent images were obtained using an inverted microscope system Eclipse Ti2 from Nikon. Limonene was stained with Nile Red $\left(\lambda_{\mathrm{ex}} \approx 550 \mathrm{~nm}, \lambda_{\mathrm{em}} \approx 630 \mathrm{~nm}\right)$. Capsules for CLSM were synthesized at $\mathrm{pH} 8$, as described above.

\section{RESULTS AND DISCUSSION}

Bulk Coacervate Properties. Ethanol-water, as the mostly used binary solvent, has the optimal condition for zein coacervate around 50\% v/v ethanol (Figure S5). Zein has different solubility in PG-water. To use simple zein coacervate in a PG-water binary solvent, we made a phase diagram to locate precipitation, coacervation, and solution regions. As Figure 1A shows, the PG-water ratio has a strong impact on
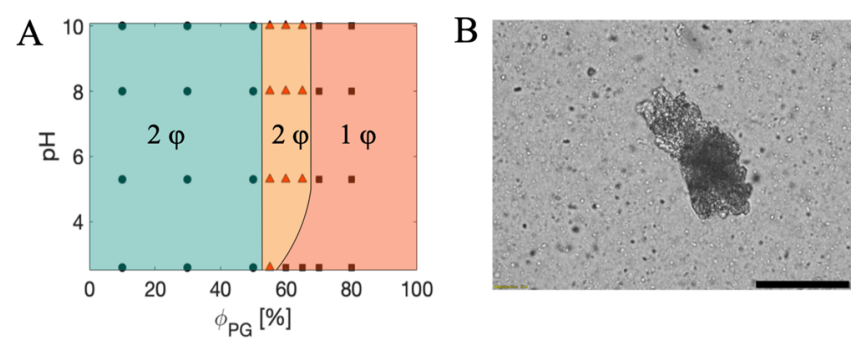

C
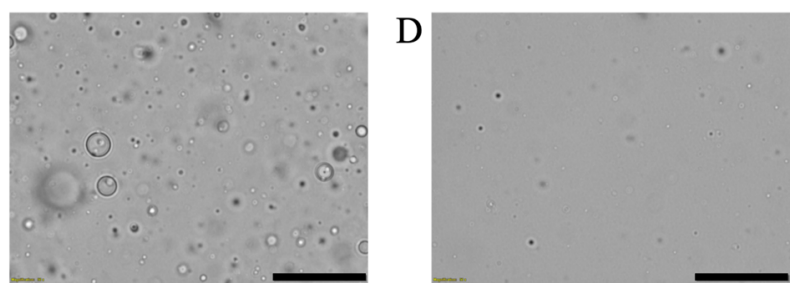

Figure 1. (A) Phase diagram for solubility of zein in propylene glycol-water binary solvents. Precipitation (circle), coacervation (triangle), and solution (square) are expressed as different symbols with the coacervate region highlighted in red. Solid lines denote the phase boundaries (note that the transitions are not very sharp). (BD) Representative optical microscopy images of zein samples at $\mathrm{pH} 8$ with different $\varphi_{\mathrm{PG}}$ of (B) $10 \%$, two-phase, precipitated region; (C) $65 \%$, two-phase, coacervated region; and (D) $80 \%$, one-phase region. Scale bars are $50 \mu \mathrm{m}$.

the solubility of zein. For a $\varphi_{\mathrm{PG}}$ between 0 and $50 \% \mathrm{v} / \mathrm{v}$, zein precipitates, and between 50 and $70 \% \mathrm{v} / \mathrm{v}$, we find a zein coacervate. Above $70 \% \mathrm{v} / \mathrm{v}$, zein becomes soluble. Representative optical microscopy images of zein samples at $\mathrm{pH} 8$ in the different regions are shown in Figure 1B-D. In part, because the zein is not completely pure, phase boundaries are not infinitely sharp, and we find narrow transition regions between the different regions (see Figures S1 and S2). For example, we find both precipitate and coacervate at $55 \% \mathrm{v} / \mathrm{v}$ for $\mathrm{pH} 2.6,5.3$, 8.0, and 10.0. In the coacervate region, the coacervate yield decreases when the solvent is approaching $70 \% \mathrm{v} / \mathrm{v}$ PG. We choose $65 \% \mathrm{v} / \mathrm{v}$ PG zein coacervates for producing capsules because, for this solvent composition, the supernatant is transparent and it has a good yield of coacervate (by visual observation). In the $\mathrm{pH}$ range that we have tested (from $\mathrm{pH}$ 2.6 to 10.0 ), we find that $\mathrm{pH}$ has only a minor effect on the solubility and phase behavior of zein. At $\mathrm{pH} 2.6$, the zein coacervate disappears when $\varphi_{\mathrm{PG}}$ is around $60 \% \mathrm{v} / \mathrm{v}$. This may be attributed to the increasing solubility as a result of the amide groups of glutamine and asparagine being hydrolyzed to carboxyl groups. ${ }^{17,22}$

Coacervate yield and protein fraction were determined at various $\mathrm{pH}$. As shown in Figure 2A, from $\mathrm{pH} 6$ to 10, the decrease of coacervate yield is not significant, but from $\mathrm{pH} 6$ to 4 , there is a clear decrease. Figure $2 \mathrm{~B}$ shows the mass fraction of protein in the coacervate. From $\mathrm{pH} 4$ to 10 , simple zein coacervate has a high protein mass fraction between 30 and $40 \%$. At $\mathrm{pH}$ lower than 4 , the protein mass fraction drops.

We measured the viscosity of zein coacervates with different $\mathrm{pH}$ at a wide range of shear rates. From Figure 3, we can see that zein coacervates have a Newtonian behavior at low shear 


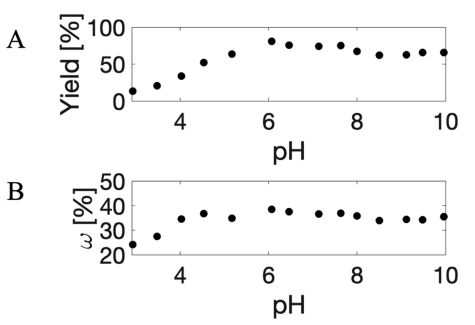

Figure 2. (A) Zein coacervate yield at $65 \% \mathrm{v} / \mathrm{v}$ PG and (B) protein mass fraction in coacervate versus $\mathrm{pH}$.

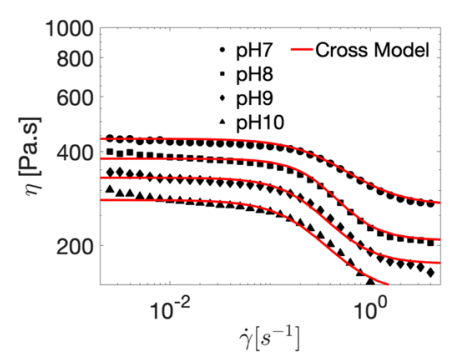

Figure 3. Viscosity of zein coacervates (produced from $65 \% \mathrm{v} / \mathrm{v}$ PG) versus shear rate. The red lines show Cross model fits. $\eta=\eta_{\infty}+\left(\eta_{0}-\right.$ $\left.\eta_{\infty}\right) /\left[1+(C \dot{\gamma})^{m}\right]$ where $\eta$ is the viscosity, $C$ is the Cross model time constant, $\dot{\gamma}$ is the shear rate, and $m$ is the Cross rate constant.

rates below $10^{-1} \mathrm{~s}^{-1}$. At higher shear rates, we observe shear thinning followed by a high shear plateau viscosity. The magnitude of the viscosity and the onset of shear thinning are both markedly influenced by $\mathrm{pH}$ : at higher $\mathrm{pH}$, when the zein molecules carry a higher net charge, viscosities are lower and shear thinning sets in at lower shear rates. Possibly, the increased net charge on the zein molecules decreases the cohesive energy of the coacervates, leading to the observed changes in the rheology. The absolute values of the viscosity that we found for the zein coacervates in the PG/water binary solvents are orders of magnitude larger than those found, for example, for gelatin-gum arabic complex coacervates ${ }^{8}$ and simple soy protein coacervates. ${ }^{23}$ At least, in part, this is caused by the much larger viscosity of the PG/water binary solvents. An empirical Cross model was used to fit the flow curves, in order to determine the zero-shear viscosities to be used to estimate interfacial tensions.

Surface Properties. We expect that the surface charge will play a significant role in determining how the coacervate droplets will coalesce. We cannot directly determine the electrophoretic mobility of coacervate droplets in $65 \% \mathrm{v} / \mathrm{v}$ PG since the coacervate droplets would have an extremely low mobility due to the high solvent viscosity, and they would most likely also coalesce and sediment during the measurements. Therefore, to obtain estimates of zein zeta potentials, we instead used zein colloids and measured their zeta potential in water at $\mathrm{pH}$ values from $\mathrm{pH} 3$ to 9.88 . Needless to say, this zeta potential may deviate from that of zein coacervate droplets in PG/water solvents, but we expect that at least the charge sign as a function of $\mathrm{pH}$ and the order of magnitude of the zeta potential should be the same. Results are shown in Figure 4. We find that the isoelectric point of zein is around $\mathrm{pH} 6.2$, in agreement with earlier results of Velikov et al. Below the isoelectric point, zein is positively charged. From $\mathrm{pH} 6.2$ to 10, the zeta potential gradually changes from 0 to $-60 \mathrm{mV}$. Below the isoelectric point, from $\mathrm{pH} 6.2$ to 4 , there is a relatively sharper increase in the zeta potential from 0 to $+60 \mathrm{mV}$. The

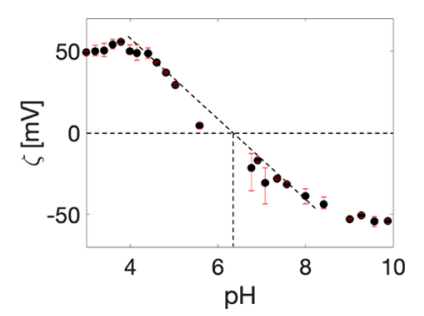

Figure 4. Zeta potential of zein colloids in water at various $\mathrm{pH}$. Error bars are indicating variations of three measurements for each sample. A straight line is used to interpolate the data for $\mathrm{pH}$ values around the isoelectric point from which we estimate an isoelectric point of $\mathrm{pI}=$ 6.2 .

absolute values of the zeta potential at $\mathrm{pH} 4(+60 \mathrm{mV})$ and 10 $(-60 \mathrm{mV})$ are very close. However, the coacervate yield at $\mathrm{pH}$ 4 is much lower than that at $\mathrm{pH} 10$. This suggests that the zein behavior is not symmetric with respect to the distance to the $\mathrm{pI}$ and that zein is more soluble in acidic conditions in the PG/ water binary solvent.

From a thermodynamic perspective, whether or not coacervate droplets wet limonene droplets in $65 \% \mathrm{v} / \mathrm{v}$ PG is determined by three interfacial tensions, as described by the spreading parameter $S^{24}$

$$
S=\gamma_{\mathrm{LP}}-\left(\gamma_{\mathrm{CP}}+\gamma_{\mathrm{CL}}\right)
$$

where $\mathrm{L}$ stands for limonene, $\mathrm{P}$ for $65 \% \mathrm{v} / \mathrm{v} \mathrm{PG}$, and $\mathrm{C}$ for coacervate. When $S>0$, coacervate droplets wet the interface completely, and when $S<0$, coacervate droplets do not wet the interface. From the pendant drop measurements shown in Figure 5, we know that $\gamma_{\mathrm{LP}}$ is between 11.5 and $12 \mathrm{mN} / \mathrm{m}$ at

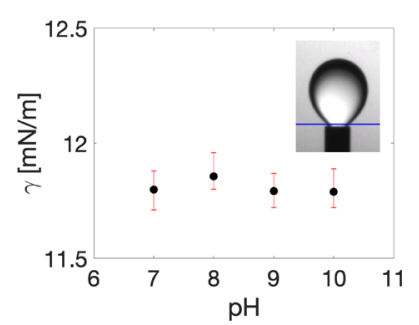

Figure 5. Interfacial tension between a limonene droplet and an aqueous PG solution $(65 \% \mathrm{v} / \mathrm{v})$ at different $\mathrm{pH}$ values. The inset figure shows the pendant drop setup with a reverse needle geometry.

$\mathrm{pH} 7,8,9$, and 10 . Due to the high viscosity of the zein coacervate and its low interfacial tension, it is experimentally more difficult to measure the interfacial tensions of zein coacervate with $65 \% \mathrm{v} / \mathrm{v}$ PG and limonene. Based on experience, the interfacial tension between coacervate and its coexisting phase is also expected to be very low. Spruijt and coworkers obtained the interfacial tension between a coacervate of two charged polyelectrolytes and its coexisting aqueous phase on the order of $100 \mu \mathrm{N} / \mathrm{m} .^{25}$ Priftis and co-workers measured the interfacial tension of polypeptide coacervates lower than $1 \mathrm{mN} / \mathrm{m}^{26}$ Low interfacial tension values $(4.2 \pm$ $0.3 \mathrm{mN} / \mathrm{m}$ ) between the coacervate and the continuous phase have also been obtained by Bago Rodriguez and co-workers through a series of calculations. ${ }^{27}$

Here, we estimate the interfacial tension $\left(\gamma_{\mathrm{CP}}\right.$ and $\left.\gamma_{\mathrm{CL}}\right)$ from capillary thinning dynamics using a method adapted from extensional rheology. ${ }^{28}$ Generally, coacervate filaments spontaneously break up in a second fluid if their configuration is out 
of the static Rayleigh-Plateau stability limit. ${ }^{29}$ This process is driven by the interplay of interfacial tension against viscous and elastic stress of the coacervate filament. To apply this method, two conditions need to be met. First, the visco-capillary time scale that determines the speed of the thinning process

$$
t=\frac{\eta_{0} d_{0}}{\gamma}
$$

needs to be experimentally accessible. In this equation, $\eta_{0}$ is the zero-shear viscosity, $d_{0}$ is the initial neck width of the filament, and $\gamma$ is the interfacial tension. This method is particularly interesting for coacervates because their low interfacial tension and their high viscosity ${ }^{25,30}$ will lead to a large visco-capillary time scale. Second, the Ohnesorge number $O h$, which balances viscous against inertial forces should be large, that is, inertia should be negligible

$$
O h=\frac{\eta_{0}}{\sqrt{\Delta \rho \gamma d_{0}}} \gg 1
$$

where $\Delta \rho$ is the buoyant density of the filament in the surrounding fluid.

Results for capillary thinning experiments are shown in Figure 6. Representative microscopy images of the thinning

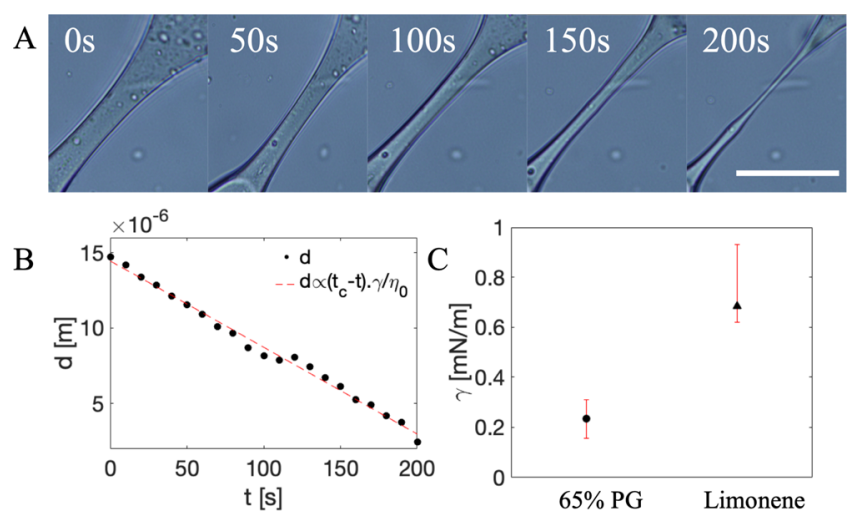

Figure 6. (A) Thinning process of a coacervate filament in $65 \% \mathrm{v} / \mathrm{v}$ PG at different time scales; the scale bar is $50 \mu \mathrm{m}$. (B) Thinning dynamics of a coacervate filament. Neck width of the filament in panel (A) as a function of time. The dashed line is a linear fit. (C) Calculated interfacial tensions of coacervate filaments in polymer depleted $65 \%$ v/v PG phase and limonene. Error bars are showing deviations among five measurements.

process for a coacervate filament in 65\% v/v PG are shown in Figure 6A. Linear regression of the thinning dynamics (neck width $d$ versus time) suggests that the coacervate filament behaves as a Newtonian fluid during thinning (Figure 6B). This is because if $\mathrm{Oh} \gg 1$, then a filament of Newtonian fluid should undergo thinning at a constant velocity of $\nu \sim \gamma / \eta_{0}$. Any elastic response of the filament would lead to thinning with a nonconstant velocity; hence, elasticity can be neglected and we can use the thinning model for a Newtonian fluid

$$
\frac{d}{d_{0}}=f \frac{\gamma}{d_{0} \eta_{0}}\left(t_{\mathrm{c}}-t\right)
$$

where $d$ is the neck width of a filament, $\gamma$ is the interfacial tension, $\eta_{0}$ is the zero-shear viscosity, and $t_{c}$ is the critical time scale for filament breakup. For the numerical constant $f$, we use $f=0.1418$ as found from Papageorgiou's similarity solution for a Newtonian fluid undergoing capillary thinning. ${ }^{31}$ We use the zero-shear viscosity, $\eta_{0}=440 \mathrm{~Pa} \cdot \mathrm{s}$ at $\mathrm{pH} 7$, as obtained from the rheology data using the Cross model fit (Figure 3). In contrast to capillary breakup extensional rheometry, where the data are fitted such that the rheological properties can be obtained for systems with a known interfacial tension, we use the inverse approach here: based on the independently measured rheological properties, we use the data here to calculate the interfacial tensions of zein coacervate in its coexisting phase $(\sim 0.24 \mathrm{mN} / \mathrm{m})$ and limonene $(\sim 0.68 \mathrm{mN} /$ $\mathrm{m})$, see Figure 6C. Replicates of the filament thinning dynamics in the excess phase or limonene oil are shown in Figures S3 and S4, respectively. Zein coacervates have a low interfacial tension with their coexisting phases and a slightly higher, but still very low, interfacial tension with the oil phase. Therefore, at $\mathrm{pH} 7$, the spreading parameter $S$ is clearly larger than zero such that complete wetting is thermodynamically favorable.

Capsule Formation. Next, we attempted to create droplets of limonene surrounded by a thick coacervate layer formed by the coalescence of coacervate droplets on the surface of the limonene droplets. First, the oil is dispersed in a one-phase zein solution at $80 \%$ PG. Coacervation is then induced by slowly adding water, thus moving the system from the one phase region into the coacervate region at $65 \%$ PG. The result of this process is shown in Figure 7 for a range of $\mathrm{pH}$ values. Starting

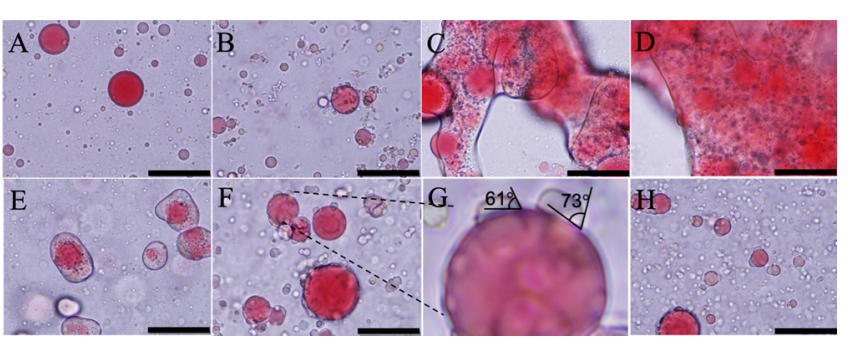

Figure 7. Light microscope pictures of capsules at (A) $\mathrm{pH} \mathrm{3,} \mathrm{(B)} \mathrm{4,}$ (C) 5, (D) 7, (E) 8, (F) 9, and (H) 10. Limonene is stained with Oil Red O. The scale bars are $50 \mu \mathrm{m}$. (G) Zoom-in image of a $\mathrm{pH} 9$ sample, showing two examples of contact angles between coacervate and oil droplets measured with ImageJ.

at a low $\mathrm{pH}$, at $\mathrm{pH} 3$, no capsules are observed, just the coexistence of oil droplets and very small coacervate droplets. At $\mathrm{pH} 4$, in addition to coacervate droplets in the bulk, we also observe coacervate droplets that have adsorbed on the oil droplets but which did not spread. Next, at $\mathrm{pH} 5$ and 7, we observe macroscopic coacervate, having engulfed almost all of the oil droplets. Moving further, at $\mathrm{pH} 8$, well-defined capsules of coacervate around oil droplets are formed. Further increasing the $\mathrm{pH}$ leads to similar behavior as observed at very low $\mathrm{pH}$ : at $\mathrm{pH} 9$ and 10, coacervate droplets attach to the surface of the oil droplets but do not spread.

The nonequilibrium nature of the spreading behavior of the coacervate is further illustrated by the zoom-in image of Figure $7 \mathrm{G}$, for $\mathrm{pH} 9$, where we show two examples of coacervate droplets attaching to the oil droplets with finite and very different contact angles $\left(61\right.$ and $73^{\circ}$ ). Clearly, even though thermodynamically favorable, the spreading of the zein coacervate around the oil droplets does not occur easily due to kinetic barriers.

Our results clearly suggest that the electrostatic repulsion between zein coacervate droplets play an important role in 
determining the kinetics of coacervate droplet attachment to and spreading on the oil droplets. For $\mathrm{pH}$ values far from the pI, the charge on the coacervate droplets prevent both attachment to the surface of the oil droplets and droplet fusion such that no capsules are formed. For $\mathrm{pH}$ values very close to the pI, on the other hand, at a low droplet charge, droplet fusion and spreading is easy and we end up with macroscopic coacervates engulfing the oil droplets. Successful capsule formation requires some droplet fusion while avoiding excessive coalescence, such that there is an optimal charge on the coacervate droplets. In principle, we would therefore expect $\mathrm{pH}$ windows for successful capsule formation on both sides of the pI. Here, we have only found such a $\mathrm{pH}$ window on the high side of the pI.

Influence of Local Shear Fields in Capsule Formation. Having established that the capsule formation is kinetically determined, we next investigate the role of local shear fields during the capsule formation process. We hypothesize that shear may aid in the coacervate droplet attachment if there are kinetic barriers and may also promote spreading. To test this hypothesis, we first produced capsules, as shown in Figure 7, using a fixed stirring speed of $430 \mathrm{rpm}$. Next, capsules were stirred 10 more minutes at the same or higher stirring rates (430, 760, and $1100 \mathrm{rpm})$. Results are shown in Figure 8.

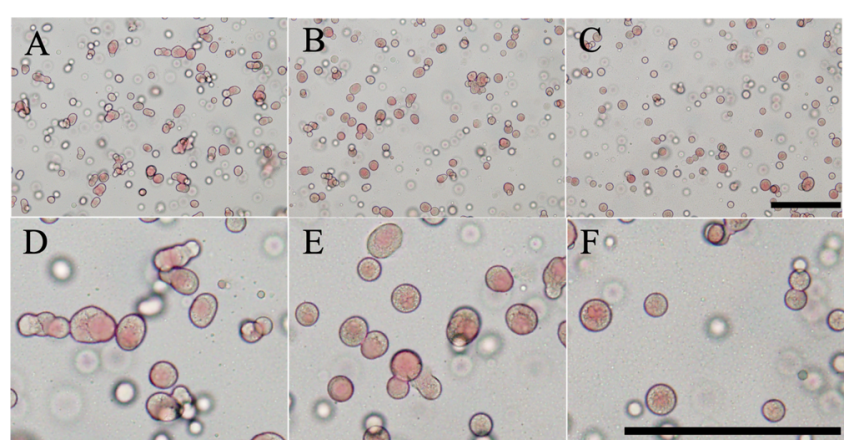

Figure 8. Light microscope pictures of capsules produced at $\mathrm{pH} 8$ at a stirring rate of $430 \mathrm{rpm}$ and further stirred for $10 \mathrm{~min}$ at the same or higher stirring rates. (A) 430, (B) 760, and (C) $1100 \mathrm{rpm}$. (D-F) Zoom-in figures of the above, respectively. Scale bars are $200 \mu \mathrm{m}$.

We find that capsules become smaller by the additional stirring at higher rates (presumably due to break up), but also more spherical, suggesting that, indeed, kinetic barriers for droplet spreading and coalescence can be overcome by shear forces.

The surface tension of the zein coacervates with the $65 \%$ PG solvent is very low (order $10^{-4} \mathrm{~N} / \mathrm{m}$ ), whereas its viscosity is very high (order $100 \mathrm{~Pa} \cdot \mathrm{s}$ ). This implies that a critical capillary number of $\mathrm{Ca} \approx 1$ corresponds to very low flow velocities of order $1 \mu \mathrm{m} / \mathrm{s}$. Hence, even low amounts of shear should, in principle, be able to deform and break the zein coacervate droplets, although the kinetics may be very slow due to the high viscosity. For limonene, on the other hand, the viscosity is much lower than that of the coacervates, and its interfacial tension with the $65 \%$ PG solvent is much higher; hence, the coacervate droplets are much more likely to be deformed by shearing than the limonene droplets.

Finally, we used CLSM to more precisely visualize the oil distribution within the capsules. Figure 9A shows a bright-field image of a capsule, showing a large oil droplet covered by coacervate. Figure 9B shows the corresponding CLSM image

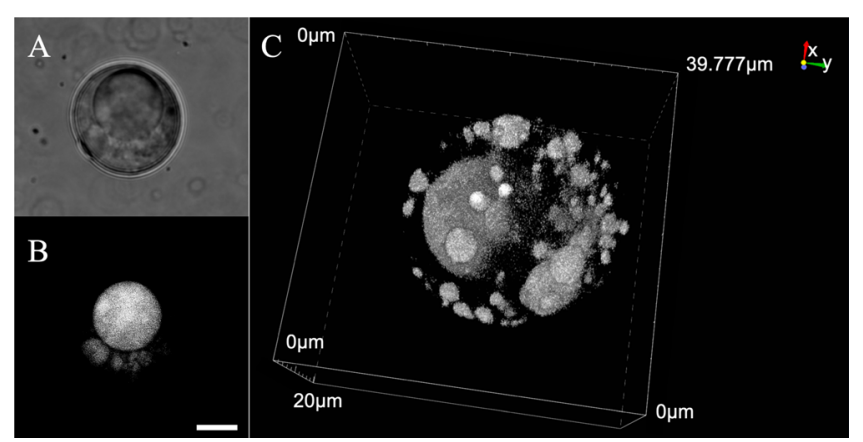

Figure 9. (A) Bright-field image of a capsule. (B) Corresponding CLSM image of the capsule in panel (A). Scale bar is $10 \mu \mathrm{m}$. (C) Three-dimensional construction of a capsule.

from which it is clear that there are in fact multiple oil cores in the capsule. Figure 9C shows a 3D construction of a capsule, indicating that this capsule contains a large oil core and some small oil droplets, which were encapsulated during coacervate coalescence. This multinuclear structure could lead to different release profiles with capsules only containing a single core. If mononucleated capsules are desired, then this could possibly be achieved by increasing the stirring rate. ${ }^{32}$

\section{CONCLUSIONS}

We have shown how simple coacervates of zein can be used to create capsules around oil droplets. As opposed to the generally much more hydrophilic animal proteins usually used to formulate complex coacervates for encapsulation, these plant proteins are quite water-insoluble and this may have advantages in terms of barrier properties. Here, we have used zein biopolymers as an example and found that, while thermodynamically, the spreading of zein coacervates droplets on oil droplets is highly favorable, the process is kinetically difficult. As a result of the high protein concentration and the high viscosity of $\mathrm{PG}$, the dynamics of coacervate droplet spreading is extremely slow. In addition, simple zein coacervate droplets carry net charges, leading to electrostatic barriers, preventing droplet attachment to the surface of the oil droplets and the fusion of coacervate droplets. Also, if the droplet charge is too low, then the rapid macroscopic phase separation of the zein ensues and no capsules are formed either.

Nevertheless, by precisely tuning the charge on the coacervate droplets via the $\mathrm{pH}$, it is possible to identify a window of solution conditions for which capsule formation is kinetically possible. We find that capsules can be formed at $\mathrm{pH}$ 8 and $65 \%$ v/v PG. Furthermore, we showed that high local shear fields can provide hydrodynamic forces to overcome some of the kinetic barriers and lead to smaller, more spherical capsules. Note that many oils (for example, soybean oil, coconut oil, and medium-chain triglycerides) are more hydrophobic than limonene. For all of these, the interfacial tension with PG-water should be higher, making the wetting process even more favorable. With limonene, we have therefore chosen a challenging case such that we expect the procedure should work for a wide range of other oils, too.

While, here, we focused on the thermodynamics and kinetics of the formation of zein coacervate layers around oil droplets, for real applications, the mechanical properties of such capsules will need to be further enhanced by cross-linking. Also, it will be very interesting to see whether, indeed, barrier properties for cross-linked zein capsules are notably different from 
capsules produced from, e.g., gelatin-gum arabic complex coacervates. ${ }^{7}$ Finally, since many plant seed storage proteins form simple coacervates, our results point to many new opportunities for using plant proteins for encapsulation.

\section{ASSOCIATED CONTENT}

\section{(s) Supporting Information}

The Supporting Information is available free of charge at https://pubs.acs.org/doi/10.1021/acsami.9b20746.

Sample vials for determining the phase diagram (Figure S1), microscopic images of the samples for determining the phase diagram (Figure S2), coacervate filament thinning dynamics in an excess phase at $\mathrm{pH} 7$ (Figure S3), $\mathrm{pH} 7$ coacervate filament thinning dynamics in limonene oil (Figure S4), and sample vials for zein in ETOH (ethanol)/water binary solvents (Figure S5) (PDF)

\section{AUTHOR INFORMATION}

\section{Corresponding Author}

Renko de Vries - Physical Chemistry and Soft Matter, Wageningen University and Research, 6708 WE Wageningen, The Netherlands; 이이.org/0000-0001-8664-3135; Email: renko.devries@wur.nl

\section{Authors}

Xiufeng Li - Physical Chemistry and Soft Matter, Wageningen University and Research, 6708 WE Wageningen, The Netherlands; (1) orcid.org/0000-0002-7615-4693

Philipp Erni - Firmenich Co, Geneva 2, Switzerland; (1) orcid.org/0000-0002-4241-0898

Jasper van der Gucht - Physical Chemistry and Soft Matter, Wageningen University and Research, 6708 WE Wageningen, The Netherlands; (1) orcid.org/0000-0001-5525-8322

Complete contact information is available at:

https://pubs.acs.org/10.1021/acsami.9b20746

\section{Notes}

The authors declare no competing financial interest.

\section{ACKNOWLEDGMENTS}

X.L., J.v.d.G., and R.d.V acknowledge financial support from Firmenich SA (grant no: OVK 16/001/20170605).

\section{REFERENCES}

(1) Liao, W. C.; Sohn, Y. S.; Riutin, M.; Cecconello, A.; Parak, W. J.; Nechushtai, R.; Willner, I. The Application of Stimuli-Responsive VEGF- and ATP-Aptamer-Based Microcapsules for the Controlled Release of an Anticancer Drug, and the Selective Targeted Cytotoxicity toward Cancer Cells. Adv. Funct. Mater. 2016, 26, $4262-4273$.

(2) de Souza, H. J. B.; de Barros Fernandes, R. V. B.; Borges, S. V.; Felix, P. H. C.; Viana, L. C.; Lago, A. M. T.; Botrel, D. A. Utility of Blended Polymeric Formulations Containing Cellulose Nanofibrils for Encapsulation and Controlled Release of Sweet Orange Essential Oil. Food Bioprocess Technol. 2018, 11, 1188-1198.

(3) Lamprecht, A.; Schäfer, U.; Leh, C. M. Influences of process parameters on preparation of microparticle used as a carrier system for $\mathrm{O}-3$ unsaturated fatty acid ethyl esters used in supplementary nutrition. J. Microencapsulation 2008, 18, 347-357.

(4) Nolles, A.; Westphal, A. H.; De Hoop, J. A.; Fokkink, R. G.; Kleijn, J. M.; Van Berkel, W. J. H.; Borst, J. W. Encapsulation of GFP in Complex Coacervate Core Micelles. Biomacromolecules 2015, 16, $1542-1549$.
(5) De Folter, J. W. J.; Van Ruijven, M. W. M.; Velikov, K. P. Oil-inWater Pickering Emulsions Stabilized by Colloidal Particles from the Water-Insoluble Protein Zein. Soft Matter 2012, 8, 6807-6815.

(6) Wang, K.; Arntfield, S. D. Binding of Carbonyl Flavours to Canola, Pea and Wheat Proteins Using GC/MS Approach. Food Chem. 2014, 157, 364-372.

(7) Dardelle, G.; Jacquemond, M.; Erni, P. Delivery Systems for Low Molecular Weight Payloads: Core/Shell Capsules with Composite Coacervate/Polyurea Membranes. Adv. Mater. 2017, 29, 1-6.

(8) Dardelle, G.; Erni, P. Three-Phase Interactions and Interfacial Transport Phenomena in Coacervate/Oil/Water Systems. Adv. Colloid Interface Sci. 2014, 206, 79-91.

(9) Zuidam, N. J.; Velikov, K. P. Choosing the Right Delivery Systems for Functional Ingredients in Foods: An Industrial Perspective. Curr. Opin. Food Sci. 2018, 21, 15-25.

(10) De Kruif, C. G.; Weinbreck, F.; De Vries, R. Complex Coacervation of Proteins and Anionic Polysaccharides. Curr. Opin. Colloid Interface Sci. 2004, 9, 340-349.

(11) Carbonaro, M.; Cappelloni, M.; Nicoli, S.; Lucarini, M.; Carnovale, E. Solubility-Digestibility Relationship of Legume Proteins. J. Agric. Food Chem. 1997, 45, 3387-3394.

(12) van Megen, W. H. Solubility Behavior of Soybean Globulins as a Function of PH and Ionic Strength. J. Agric. Food Chem. 1974, 22, 126-129.

(13) Nesterenko, A.; Alric, I.; Silvestre, F.; Durrieu, V. Vegetable Proteins in Microencapsulation: A Review of Recent Interventions and Their Effectiveness. Ind. Crops Prod. 2013, 42, 469-479.

(14) Liu, F.; Tang, C. H. Soy Protein Nanoparticle Aggregates as Pickering Stabilizers for Oil-in-Water Emulsions. J. Agric. Food Chem. 2013, 61, 8888-8898.

(15) Liang, H. N.; Tang, C. Pea Protein Exhibits a Novel Pickering Stabilization for Oil-in-Water Emulsions at PH 3.0. LWT - Food Sci. Technol. 2014, 58, 463-469.

(16) Li, X.; de Vries, R. Interfacial Stabilization Using Complexes of Plant Proteins and Polysaccharides. Curr. Opin. Food Sci. 2018, 21, $51-56$.

(17) Shukla, R.; Cheryan, M. Zein: the Industrial Protein from Corn. Ind. Crops Prod. 2001, 13, 171-192.

(18) Filippidi, E.; Patel, A. R.; Bouwens, E. C. M.; Voudouris, P.; Velikov, K. P. All-Natural Oil-Filled Microcapsules from WaterInsoluble Proteins. Adv. Funct. Mater. 2014, 24, 5962-5968.

(19) Dai, L.; Sun, C.; Wei, Y.; Mao, L.; Gao, Y. Characterization of Pickering Emulsion Gels Stabilized by Zein/Gum Arabic Complex Colloidal Nanoparticles. Food Hydrocolloids 2018, 74, 239-248.

(20) Lazko, J.; Popineau, Y.; Legrand, J. Soy Glycinin Microcapsules by Simple Coacervation Method. Colloids Surf., B 2004, 37, 1-8.

(21) Lawton, J. W. Zein: A History of Processing and Use. Cereal Chem. 2002, 79, 1-18.

(22) Li, Y.; Li, J.; Xia, Q.; Zhang, B.; Wang, Q.; Huang, Q. Understanding the Dissolution of $\alpha$-Zein in Aqueous Ethanol and Acetic Acid Solutions. J. Phys. Chem. B 2012, 116, 12057-12064.

(23) Lui, D. Y. M.; Litster, J. D.; White, E. T. Precipitation of Soy Proteins : Particle Formation and Protein Separation. AIChE J. 2007, 53, 2-10.

(24) Torza, S.; Mason, S. G. Three-Phase Interactions In Shear and Electrical Fields. J. Colloid Interface Sci. 1970, 33, 67-83.

(25) Spruijt, E.; Sprakel, J.; Cohen Stuart, M. A.; Van Der Gucht, J. Interfacial Tension between a Complex Coacervate Phase and Its Coexisting Aqueous Phase. Soft Matter 2010, 6, 172-178.

(26) Priftis, D.; Farina, R.; Tirrell, M. Interfacial Energy of Polypeptide Complex Coacervates Measured via Capillary Adhesion. Langmuir 2012, 28, 8721-8729.

(27) Bago Rodriguez, A. M.; Binks, B. P.; Sekine, T. Emulsion Stabilisation by Complexes of Oppositely Charged Synthetic Polyelectrolytes. Soft Matter 2018, 14, 239-254.

(28) McKinley, G. H. Visco-Elasto-Capillary Thinning and Break-Up of Complex Fluids. Annu. Rheol. Rev. 2005, 1-49. 
(29) Mora, S.; Phou, T.; Fromental, J. M.; Pismen, L. M.; Pomeau, Y. Capillarity Driven Instability of a Soft Solid. Phys. Rev. Lett. 2010, 105, 1-4.

(30) Liu, Y.; Winter, H. H.; Perry, S. L. Linear Viscoelasticity of Complex Coacervates. Adv. Colloid Interface Sci. 2017, 239, 46-60.

(31) Papageorgiou, D. T. On the Breakup of Viscous Liquid Threads. Phys. Fluids 1995, 7, 1529.

(32) Lemetter, C. Y. G.; Meeuse, F. M.; Zuidam, N. J. Control of the Morphology and the Size of Complex Coacervate Microcapsules during Scale-Up. AIChE J. 2009, 55, 1487-1496. 